\title{
ANÁLISIS ESPACIO-TEMPORAL (1956-2004) DE LOS SISTEMAS DUNARES DE MENORCA (ISLAS BALEARES) MEDIANTE VARIABLES GEOAMBIENTALES DE USO Y GESTIÓN
}

\author{
F.X. Roig-Munar( ${ }^{(1,2)}$, G.X. Pons ${ }^{(2)}$, J.A. Martín-Prieto(1, 2), A. Rodríguez-Perea ${ }^{(2)}$ y M. Mir-Gual(2) \\ (1) QU4TRE, consultoria ambiental \\ (2) Departamento de Ciències de la Terra, Universitat de les Illes Balears
}

\section{RESUMEN}

En este trabajo se analizan 28 sistemas playa-duna de la isla de Menorca (Islas Baleares) mediante variables de uso, estado y gestión a lo largo de 6 períodos, comprendidos entre 1956 y 2004. Los resultados obtenidos mediante el análisis multiparamétrico durante este periodo ha permitido obtener una visión particular y de conjunto sobre la evolución geoambiental de los sistemas playa-duna analizados, y una clasificación de éstos. Ambos resultados están directamente relacionados con las medidas de gestión aplicadas sobre cada uno de los sistemas.

Palabras clave: playa-duna, Menorca, variables, clasificación, evolución, medidas de gestión.

\begin{abstract}
Throughout 6 periods among 1956 and 2004, 28 beach - dune systems of the island of Menorca (Balearic island.) are analyzed by means of variables of use, condition state and management. The results obtained by means of the multiparametric analysis in time has facilitated a detailed interpretation which is helpful to understand the geoambiental evolution of the analyzed beach - dune systems as well as a classification of them. Both outputs are directly related with the management that these systems hav undergone.
\end{abstract}

Fecha de recepción: octubre 2009.

Fecha de aceptación: febrero 2012. 
Key words: Menorca, beach-dune, variables, classification, evolution, conservation and management.

\section{INTRODUCCIÓN}

En términos generales la ocupación del litoral por parte del hombre ha sido masiva, rápida y acultural, dándose un proceso de litoralización acelerado. Muchas de estas ocupaciones y transformaciones han dado lugar a la interrupción de mecanismos naturales de formación y defensa, impidiendo el buen funcionamiento de los ecosistemas litorales (Brown y McLachlan, 1990), y modificado notablemente el paisaje, dando lugar a cambios en sus características naturales y tradicionales (Pinto, 2005). Este cambio ha sido imprevisto, en la medida que al inicio del desarrollo turístico no se concibieron los espacios litorales - las playas y las dunas - como espacios frágiles y dinámicos susceptibles a degradarse rápidamente. En este sentido Nordstrom (1994) hace referencia a la gestión sobre el medio litoral como una acción aberrante, reclamando que las acciones que se realicen sean integrales y no como intromisión a los procesos naturales. Por su parte Bejarano et al. (1997) argumentan que las agresiones sobre el litoral se encuentran disfrazadas como «factores socioeconómicos» que han permitido la rotura de sistemas y ámbitos de riqueza natural, como es el caso de los sistemas dunares. Esta interacción de factores ambientales que se da en los ámbitos litorales determina una gran variedad de procesos que hace de estos espacios áreas de elevada fragilidad geoambiental.

En el caso de Menorca el litoral constituye el principal activo medioambiental sobre el que se basa la economía de la isla. Estos espacios litorales pertenecen a complejos sistemas sobre los que intervienen gran número de factores (Martín-Prieto y Rodríguez-Perea, 1996), de diferente naturaleza y magnitud, y su preservación está directamente relacionada con la posibilidad de adaptación natural a cambios dinámicos provocados por agentes de carácter natural y/o antrópico.

En el caso de los sistemas dunares, estos forman parte de un sistema mas complejo denominado sistema playa-duna, caracterizado por la interacción de dos ámbitos diferenciados; el ámbito sumergido, controlado por la hidrodinámica marina, y el ámbito emergido, controlado por dinámica eólica. Estos sistemas playa-duna representan un sistema frágil en su conjunto, ya que las pequeñas modificaciones pueden romper su equilibrio natural en cada uno de sus ámbitos. Esta fragilidad ha comportado que en zonas eminentemente turísticas hayan sufrido procesos de modificación, regresión, y en los peores casos su desaparición, debido a su urbanización y a la falta de una adecuada gestión, mantenimiento y preservación.

A partir de la década de los 70 hasta la actualidad, la gestión litoral ha estado marcada por su explotación, por la preocupación de cumplir con las normas básicas de higiene y por la instalación de ciertos servicios destinados al ocio y recreación de usuarios, no concibiendo estos espacios como sistemas naturales. Las playas y calas fueron entendidas como un anexo más a los espacios urbanos y turísticos, obviando sus características ambientales, las funciones de reserva sedimentológica de las morfologías dunares asociadas, y el hecho que constituyen el hábitat de numerosas especies botánicas adaptadas a estos ambientes, e imprescindibles para el mantenimiento y estabilización de los sistemas (Schmitt, 1994). 
En general la instalación de servicios de playa de forma indiscriminada y aleatoria sobre las foredunes y las dunas ha contribuido a la generación de diversas morfologías erosivas (Alonso et al., 2002), sin que hayan sido objeto de restitución. Estos procesos de alteración y degradación, a los que Hesp $(1988,2002)$ clasificó morfoecológicamente, ejemplifican los estadios de estabilidad y erosión y su posible recuperación, basada esta en su frente dunar, siendo este frente el que da estabilidad al conjunto del sistema.

Los sistemas dunares responden a procesos externos y a factores ambientales que actúan directamente sobre ellos. Los procesos externos que intervienen son los que determinan la incidencia de los factores ambientales, responsables de la ralentización de sus procesos de formación y desequilibrio. Estos se pueden enmarcar en dos elementos perturbadores: asociados a factores ambientales; o bien relacionados con factores de gestión y uso, que determinaran la dinámica actual de cada uno de los sistemas con uso turístico y/o recreativo. Las actividades de presión antrópica, relacionadas con la recreación y el ocio, tienen su punto de partida en el reciente desarrollo turístico del litoral. Estas se pueden agrupar en dos grupos:

1. Actividades que implican la destrucción del sistema y/o urbanización parcial o total del sistema. Estas actuaciones provocan importantes desequilibrios de forma rápida y acelerada.

2. Las actuaciones de gestión y uso, que son aparentemente menos destructivas que las primeras, pero más sistemáticas y extensas, y engloban buena parte de las actividades que se dan sobre el sistema. Tanto las actividades de uso como las de gestión y la instalación de servicios degradan el conjunto del sistema. Las zonas más afectadas por este tipo de impactos son las zonas de mayor uso, las zonas de playa y foredune, siendo también las más frágiles y de trascendencia dinámica, tanto hacia el interior del sistema como en la parte alta de la playa. Será el estado de estas morfologías delanteras las que contribuirán a la estabilización o degradación del sistema en todo su conjunto.

En el caso de Menorca el primer análisis geoambiental de sistemas dunares fue realizado por Rita et al. (1988). Este trabajo analizaba 7 playas, con aportación de cartografía temática en la que se reflejaban las presiones a que estaban sometidas y las tendencias observadas. Posteriormente, Servera (1997) realizó una descripción geomorfológica de 13 sistemas playa-duna, apuntando en cada uno de ellos las causas de la regresión a la que estaban sometidos. Cardona et al. (2004) analizan la evolución histórica de 9 sistemas dunares, comparando los impactos desde 1988 hasta 2002, y tomando como referencia el trabajo de Rita et al. (1988). Los tres trabajos citados ya hacían referencia a estados de degradación acelerados e incipientes en algunos de los arenales, debido al uso y gestión. A pesar de que estos trabajos fueron pioneros en el análisis de los sistemas playa-duna de Menorca, se limitaron a las grandes unidades, dejando 15 playas o calas sin analizar o describir, todas ellas de menor escala.

\section{HIPÓTESIS Y OBJETIVOS}

Debido que Menorca es la isla de las Baleares que cuenta con mayor número de sistemas dunares, y donde su estado de conservación va desde espacios bien conservados a mal conservados. Dado que la dinámica que se ha dado sobre estos puede ser evaluable con el uso de distintas variables espacio-temporales. Ello permite realizar un análisis con el que poder 
apreciar las respuestas geoambientales del los sistemas, asociadas a sus medidas de uso y gestión. De este modo se puede obtener como resultado un modelo evolutivo a lo largo de los períodos analizados.

El estudio parte de la premisa que el estado de conservación de cada uno de los sistemas en 1956 (fecha de la primera referencia de fotografías aéreas) era óptimo. En ese momento se considera un supuesto estado de equilibrio en el conjunto del sistema playa-duna, con una estructuración vegetal y morfológica en su frente dunar, clasificada según Hesp (2002), como estadio 1, y una zonación desde la zona de playa emergida hasta las zonas de dunas estabilizadas equilibrada, en donde los únicos agentes erosivos que podían afectar al sistema eran los de origen natural, siendo la respuesta del sistema la recuperación progresiva y natural de estos (Pye, 1982). Este estado de equilibrio obedece al hecho que Menorca no sufría en los años 50-60 la presión turística recreativa en su litoral.

Tomamos como base de partida los estudios realizados en Baleares por Roig-Munar y Comas (2005), los cuales realizaron un análisis de clasificación de playas y calas de las islas mediante el uso de variables, la propuesta de un modelo de clasificación para su gestión, y un modelo de evolución temporal de estas. Este análisis se realizó con los datos de campo de 2003 y 2004, obteniendo una radiografia estática de la situación actual de la oferta de playas de las Islas Baleares, siendo esta metodología una herramienta de gestión para futuras actuaciones sobre cada unidad analizada.

LOCALIZACIÓN DE LOS 28 SISTEMAS PLAYA-DUNA DE LA ISLA DE MENORCA (ISLAS BALEARES)

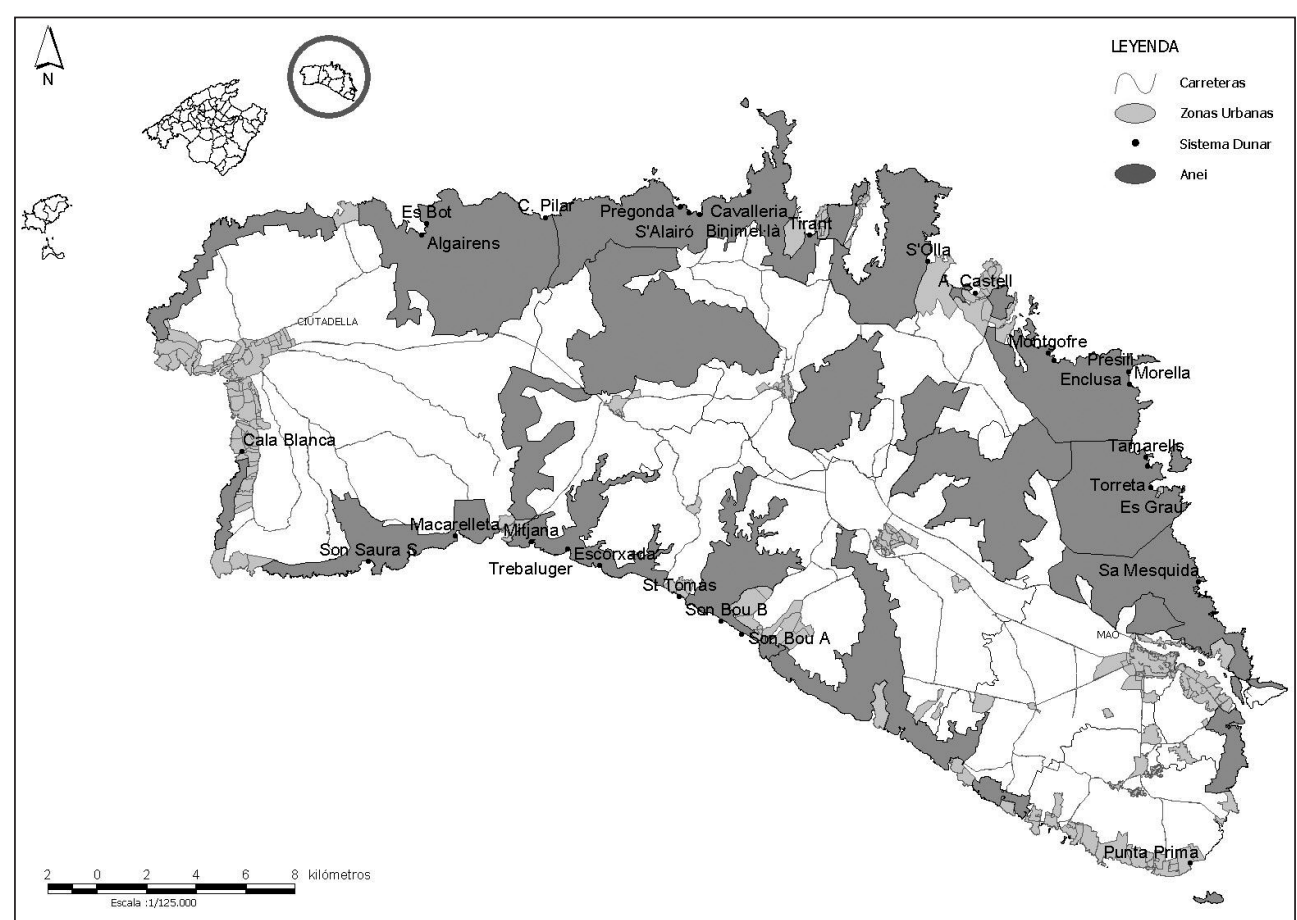


Tabla 1

RELACIÓN DE SISTEMA DUNARES DE MENORCA, SEGÚN LA CLASIFICACIÓN DE GESTIÓN ESTABLECIDA POR ROIGMUNAR (2003)

\begin{tabular}{|c|c|c|}
\hline SISTEMAS DE TIPOLOGÍA A & SISTEMAS DE TIPOLOGÍA B & SISTEMAS DE TIPOLOGÍA C \\
\hline ARENAL CASTELL & BINIMEL·LÀ & CALA PILAR \\
\hline CALA BLANCA & CAVALLERIA & ESCORXADA \\
\hline PUNTA PRIMA & ES BOT & MORELLA \\
\hline SANT ADEODATO & ES GRAU & S'ENCLUSA \\
\hline SANT TOMÀS & ES TANCATS & TAMARELLS \\
\hline SON BOU A & MACARELLETA & TORRETA \\
\hline S'OLLA (SON SAURA N) & MESQUIDA & \\
\hline & MITJANA & \\
\hline & PREGONDA & \\
\hline & S'ALAIRÓ (PREGONDÓ) & \\
\hline & SON BOU B & \\
\hline & SON SAURA S & \\
\hline & TIRANT & \\
\hline & & \\
\hline & &
\end{tabular}

Por tanto, el objetivo del presente trabajo es el de realizar un análisis espacio temporal a macro escala (Larson y Graus, 1995), de la evolución de 28 sistemas playa-duna de Menorca (Figura 1), todos ellos de diferente clasificación según su uso de variables geoambientales de gestión y conservación (Tabla 1). Se escogen estos sistemas dunares por ser ambientes frágiles y dinámicos, sobre los cuales se pueden apreciar las tendencias que se dan, ya sean erosivas, de estabilidad o de recuperación, en forma de respuestas morfológicas apreciables a lo largo del tiempo. Su conocimiento ayudará a establecer un posible modelo sobre su situación actual y las tendencias de los sistemas.

\section{METODOLOGÍA}

Para el estudio se ha establecido la siguiente metodología, basada en la adaptación y modificación de diferentes métodos consultados bibliográficamente y adaptados a las condiciones y realidades de la isla de Menorca:

1. Selección representativa y exhaustiva de sistemas playa-duna de Menorca: 28 casos de estudio (Figura 1). El sistema de Son Bou se ha dividido en dos unidades (A y B (Tabla 1)) ya que el estado geoambiental y el uso que se ha dado sobre el sistema a lo largo del tiempo de análisis han sido muy distintos, y como consecuencia se han considerado dos unidades morfoecológicas bien diferenciadas (Roig-Munar, 2004).

2. Determinación de los períodos de análisis. Nos hemos basado en los períodos 1956, 1975, 1983 , 1995, 2000 y 2004, a partir de las fotografías aéreas para poder observar los cambios más significativos de cada unidad (Crowell et al., 1991; Valpreda y 


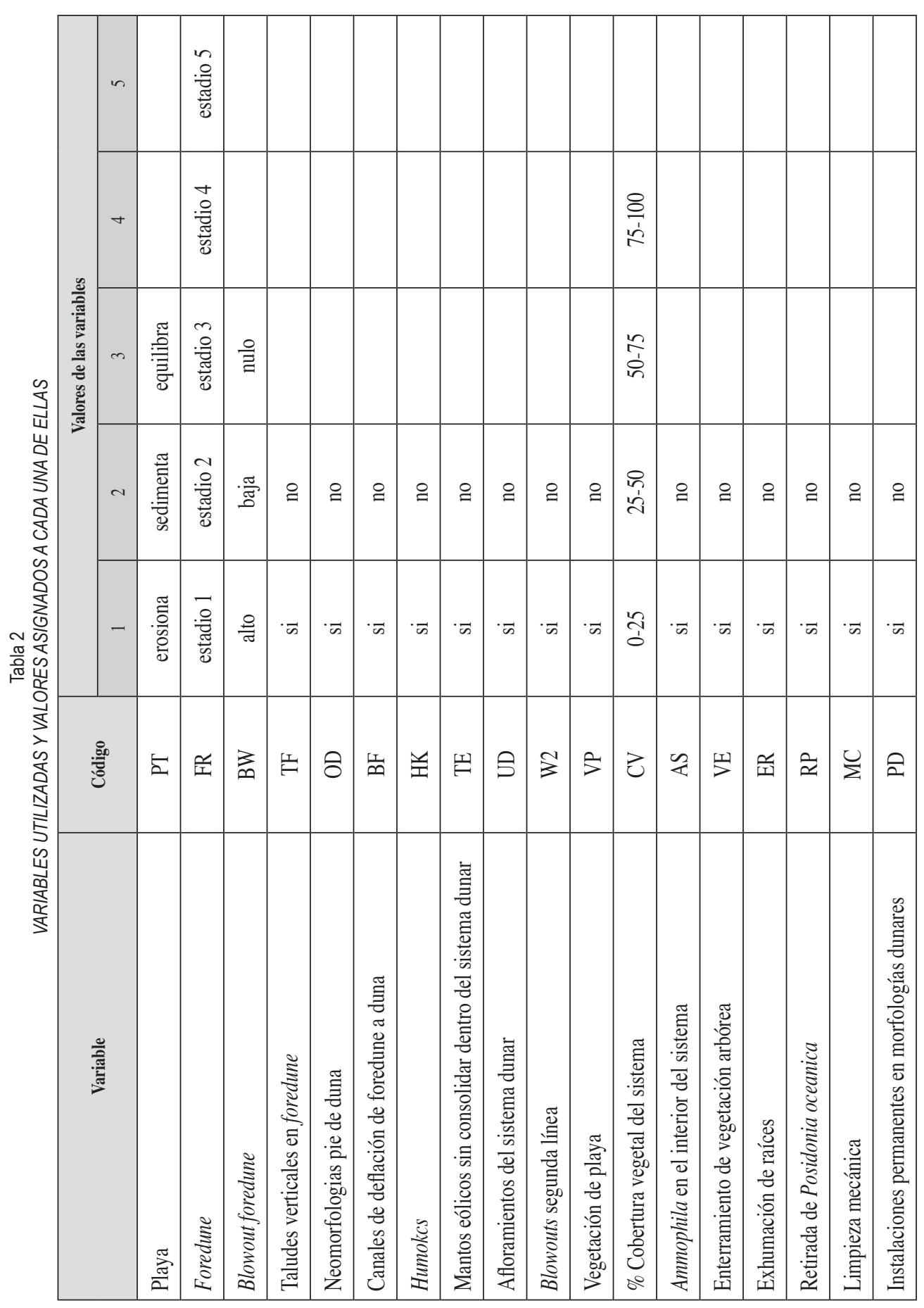




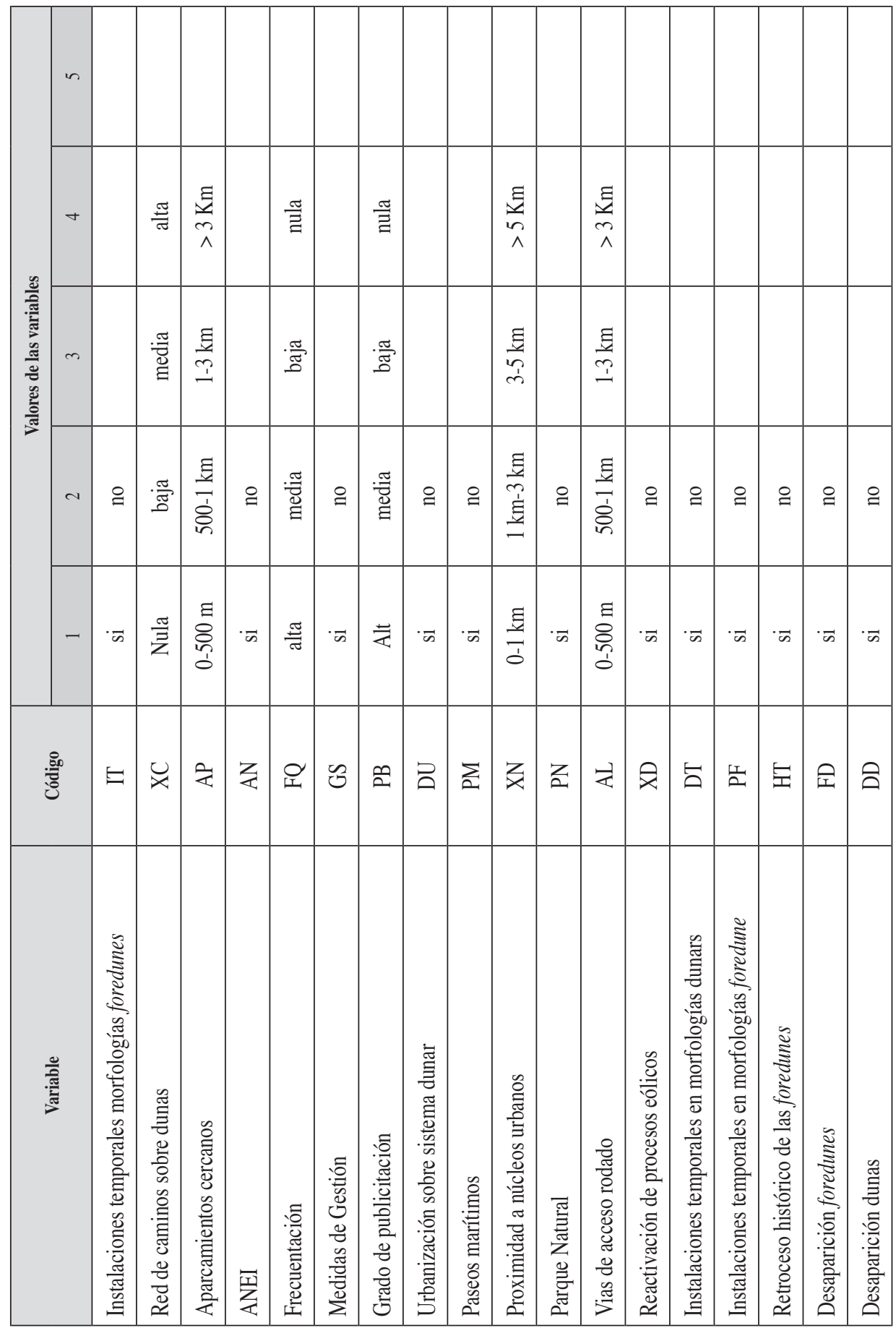


Simeoni, 2003), añadiendo una componente temporal y evolutiva al análisis del sistema (Garrote y Garzón, 2004).

3. Se han identificado y definido un grupo representativo de 36 variables para su posterior análisis de ordenación, utilizando parámetros físicos y ambientales, para establecer una tendencia evolutiva desde el año 1956 hasta 2004 (García et al., 2001; McLaughlin et al., 2002; Hesp, 1998 y 2002), y parámetros de estado, uso y gestión (Williams y Davies, 1997; Williams y Morgan, 1995; Leatherman, 1997; Laranjerira et al., 1999; Roig-Munar y Comas, 2005). Estas 36 variables (Tabla 2) son de tipo cualitativo, mayoritariamente discontinuas con valores comprendidos entre 0 y 5 , y tienen como objetivo la caracterización del estado de los sistemas dunares. Se han asignado valores para cada una de las variables y cada caso de estudio mediante análisis fotogramétrico, consulta bibliográfica, fuentes orales de antiguos responsables municipales de gestión de playas y los últimos años trabajo de campo.

Estas 36 variables son de tipo:

- Geomorfológicas (19); sistema playa, estado de las foredunes (Hesp, 2002), presencia de blowouts, taludes erosivos de foredune, neomorfologías de pie de duna, canales de deflación, presencia de hummocks, mantos eólicos sin consolidar, desaparición de foredunes, retroceso histórico de foredunes, desaparición de dunas, afloramientos rocosos en el sistema dunar, blowouts en segunda línea del sistema, vegetación de playa, porcentaje de cobertura vegetal, Ammophila arenaria dentro del sistema, enterramiento de vegetación, exhumación de raíces de porte arbóreo, y reactivación de procesos eólicos dentro del sistema dunar.

- De uso (8); densidad de caminos sobre el sistema dunar (Voigt y Weidemann 1997; Curr et al., 2000), distancia de los aparcamientos, distancia de las vías de acceso, grado de frecuentación, grado de publicitación (Blázquez y Roig-Munar, 1999), presencia de urbanización sobre el sistema, paseo marítimo, y proximidad de núcleos urbanos y/o turísticos.

- De gestión (9); retirada de acumulaciones de Posidonia oceanica, limpiezas mecánicas (Roig-Munar, 2004), instalaciones permanentes, y instalaciones temporales sobre morfologías dunares, instalaciones permanentes, y instalaciones temporales sobre foredunes (Alonso et al., 2002), Área Natural d'Especial Interés (ANEI), Parque Natural, y medidas de gestión, protección y recuperación.

4. Análisis de redundancia mediante el cálculo de una matriz de correlaciones sobre las 36 variables iniciales, para seleccionar una submatriz con variables no redundantes (Tabla 3).

5. Análisis de Componentes Principales (ACP) sobre una matriz definida por 168 casos (28 unidades de análisis por 6 períodos de estudio) y 15 variables no redundantes (Tabla 3).

6. Tendencias observadas en los períodos de análisis, y propuesta de un modelo de clasificación de sistemas playa-duna en el espacio-tiempo. 
Tabla 3

RELACIÓN DE 15 VARIABLES NO REDUNDANTES MEDIANTE EL ANÁLISIS DE REDUNDANCIA

\begin{tabular}{|l|c|}
\hline \multicolumn{1}{|c|}{ VARIABLES NO REDUNDANTES } & CÓDIGO \\
\hline 1. Playa & PT \\
\hline 2. Foredune & FR \\
\hline 3. Neomorfologías en pie de duna & OD \\
\hline 4. Canales de deflación de foredune a duna & BF \\
\hline 5. Afloramientos en el sistema dunar & UD \\
\hline 6. Vegetación de playa & VP \\
\hline 7. Porcentaje de cobertura vegetal & CV \\
\hline 8. enterramiento de vegetación arbórea & VE \\
\hline 9. Exhumación de raíces & ER \\
\hline 10. Instalaciones permanentes morfologías dunars & PD \\
\hline 11. ANEI & AN \\
\hline 12. Medidas de gestión & GS \\
\hline 13. Urbanización sobre el sistema dunar & DU \\
\hline 14. Parque Natural & PN \\
\hline 15. Instalaciones permanentes morfologías foredune & PF \\
\hline
\end{tabular}

\section{RESULTADOS}

El Análisis de Componentes Principales (SPSS v.12) sobre una matriz de 168 casos y 15 variables no redundantes extrae 4 factores significativos, que explican conjuntamente el $62,3 \%$ de la varianza (Tabla 4), y los coeficientes de correlación de las variables extraídas del ACP (Tabla 5).

Los tres primeros factores explican más de la mitad de la varianza (53,5\%). El factor F1 (Figura 2) está altamente y positivamente correlacionado con las variables DU (urbanización) y PF (instalaciones en foredune), y negativamente se correlaciona con parámetros

Tabla 4

VARIANZA TOTAL EXPLICADA POR LOS FACTORES SIGNIFICATIVOS EXTRAÍDOS DEL ANÁLISIS DE LOS COMPONENTES PRINCIPALES

\begin{tabular}{|c|c|c|c|}
\hline Factor & Total & \% de Varianza & \% Acumulado \\
\hline F1 & 4,091 & 27,276 & 27,276 \\
\hline F2 & 2,286 & 15,240 & 42,516 \\
\hline F3 & 1,646 & 10,971 & 53,487 \\
\hline F4 & 1,323 & 8,822 & 62,308 \\
\hline
\end{tabular}


Tabla 5

COEFICIENTES DE CORRELACIÓN DE LAS VARIABLES CON LOS FACTORES EXTRAÍDOS DEL ACP

\begin{tabular}{|c|c|c|c|c|}
\hline Variable & $\mathbf{F 1}$ & $\mathbf{F 2}$ & $\mathbf{F 3}$ & F4 \\
\hline PT & 0,581 & 0,207 & $-0,164$ & $-0,039$ \\
\hline FR & $\mathbf{- 0 , 7 6 4}$ & $-0,148$ & 0,030 & 0,090 \\
\hline OD & $\mathbf{- 0 , 7 2 5}$ & 0,032 & 0,236 & 0,477 \\
\hline BF & 0,524 & 0,265 & 0,219 & $-0,208$ \\
\hline UD & 0,369 & 0,500 & 0,052 & 0,131 \\
\hline VP & $\mathbf{- 0 , 6 0 7}$ & 0,099 & 0,156 & 0,563 \\
\hline CV & 0,546 & $-0,273$ & 0,519 & 0,054 \\
\hline VE & 0,325 & 0,287 & 0,680 & 0,097 \\
\hline ER & 0,423 & 0,417 & 0,455 & 0,184 \\
\hline PD & 0,547 & $-0,337$ & $-0,296$ & 0,542 \\
\hline AN & $-0,012$ & $\mathbf{0 , 6 4 2}$ & $-0,571$ & 0,001 \\
\hline GS & 0,126 & $\mathbf{0 , 6 4 9}$ & $-0,179$ & 0,018 \\
\hline DU & $\mathbf{0 , 7 5 8}$ & $-0,216$ & $-0,165$ & 0,262 \\
\hline PN & $-0,125$ & $\mathbf{0 , 6 4 2}$ & $-0,100$ & 0,368 \\
\hline PF & $\mathbf{0 , 6 1 4}$ & $-0,391$ & $-0,256$ & 0,406 \\
\hline
\end{tabular}

geomorfológicos (FR (foredune) y OD (neomorfologías)) y botánicos (VP). Teniendo en cuenta que la mayoría de las 15 variables analizadas toman valores crecientes en el sentido 'presencia - ausencia', se entiende que tienen una significación inversa; es decir, que los valores máximos (5) se corresponden con la ausencia del parámetro medido (DU= 5 indica nula urbanización). En consecuencia, la alta correlación de F1 con DU y PF equivale a una elevada correlación con lo «no urbanizado» y con la ausencia de instalaciones en morfologías foredune. Así, se interpreta a F1 como una medida inversa al grado de alteración de la parte frontal de los sistemas dunares, es decir, como una medida del grado de conservación o alteración, - según los valores establecidos por Hesp (2002) - , que en muchos casos dependerá de la gestión efectuada (Brown y McLachlan, 1998, Roig-Munar et al., 2004), o de la gestión y control del uso público sobre estas morfologías. La alteración del frente dunar, ya sea debido a su uso y/o gestión, o por procesos naturales puede determinar el estado de conservación en el conjunto del sistema, dándose procesos erosivos con base en su frente y programación hacia el sistema interior, mediante canales de deflación, que se agravaran por la falta de gestión o por una mala gestión de estos espacios.

El factor F2 muestra una correlación elevada con las figuras de protección (PN (parque natural) y AN (ANEI)) y las medidas de gestión (GS). Debido a que las variables toman valores inversos, hay que entender este factor como un indicador inverso a la presencia de actuaciones encaminadas a su conservación: valores F2 negativos indican que se han 
adoptado medidas de gestión (regulación de actividades, gestiones de tipo morfológico y regulación del uso público) y protección, aunque estas medidas de protección, mediante la aprobación de Ley Autonómica de Espacios Naturales (LEN) 1/1991, que declaraba la figura de Áreas Naturales de Especial Interés (ANEI), y que otorgaba protección urbanística a un elevado porcentaje litoral de Menorca, no incidía sobre las medias de protección de los medios litorales. El resto de factores presentan correlaciones muy débiles, siendo F3 correlaciones negativas con AN y PD, y positivas con VE y CV, por lo que se interpreta como una medida en el grado de cobertura vegetal del sistema. F4 se correlaciona negativamente con $\mathrm{BF}$ y positivamente con OD, PD y VP, y F5 muestra correlaciones negativas con BF y CV y positivas con VE y PT.

Figura 2

CORRELACIÓN DE LAS 15 VARIABLES CON LOS FACTORES F1 Y F2 EXTRAÍDOS DEL ACP

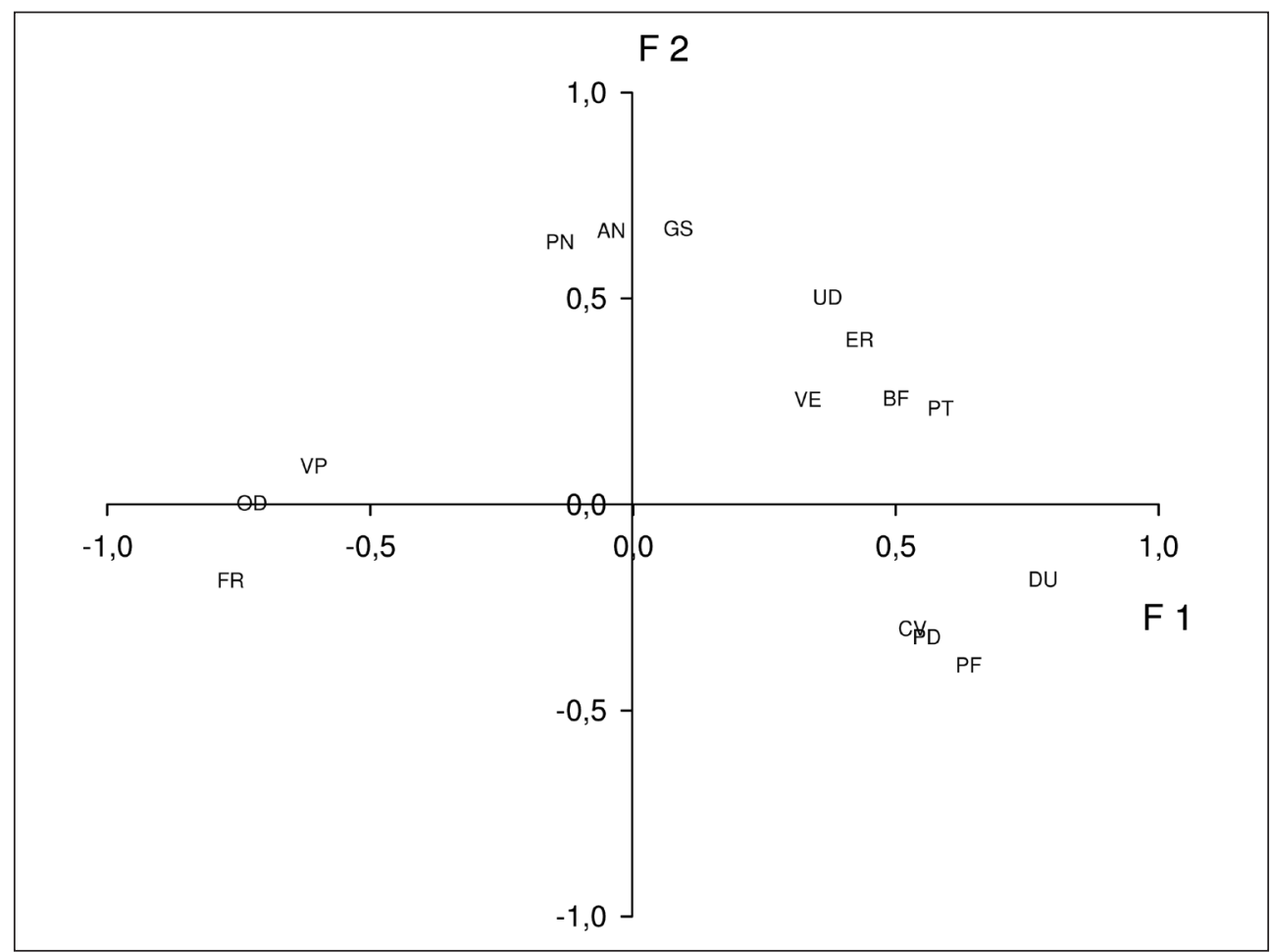

De este modo tenemos que los componentes de los factores F1 y F2 definen un espacio factorial con 4 cuadrantes bien definidos (Figura 3), siendo estos:

1. Sistemas no alterados ni gestionados. No existe uso, protección ni gestión del medio litoral arenoso. Por tanto estamos ante una fase de naturalidad del sistema que se regenera de forma natural ante procesos erosivos puntuales de tipo natural (F1 y F2 positivos). 
2. Sistemas alterados o en proceso de alteración, no se dan procesos gestión ni protección y los sistemas dunares empiezan una tendencia hacia la degradación sistemática (F1 negativo y F2 positivo).

3. Sistemas alterados, pero gestionados, los procesos erosivos se ralentizan o estabilizan sin que haya la aplicación de métodos o técnicas de recuperación de la forma erosionada (F1 y F2 negativos), y

4. Sistemas no alterados o recuperados, y gestionados y protegidos (F1 positivo y F2 negativo).

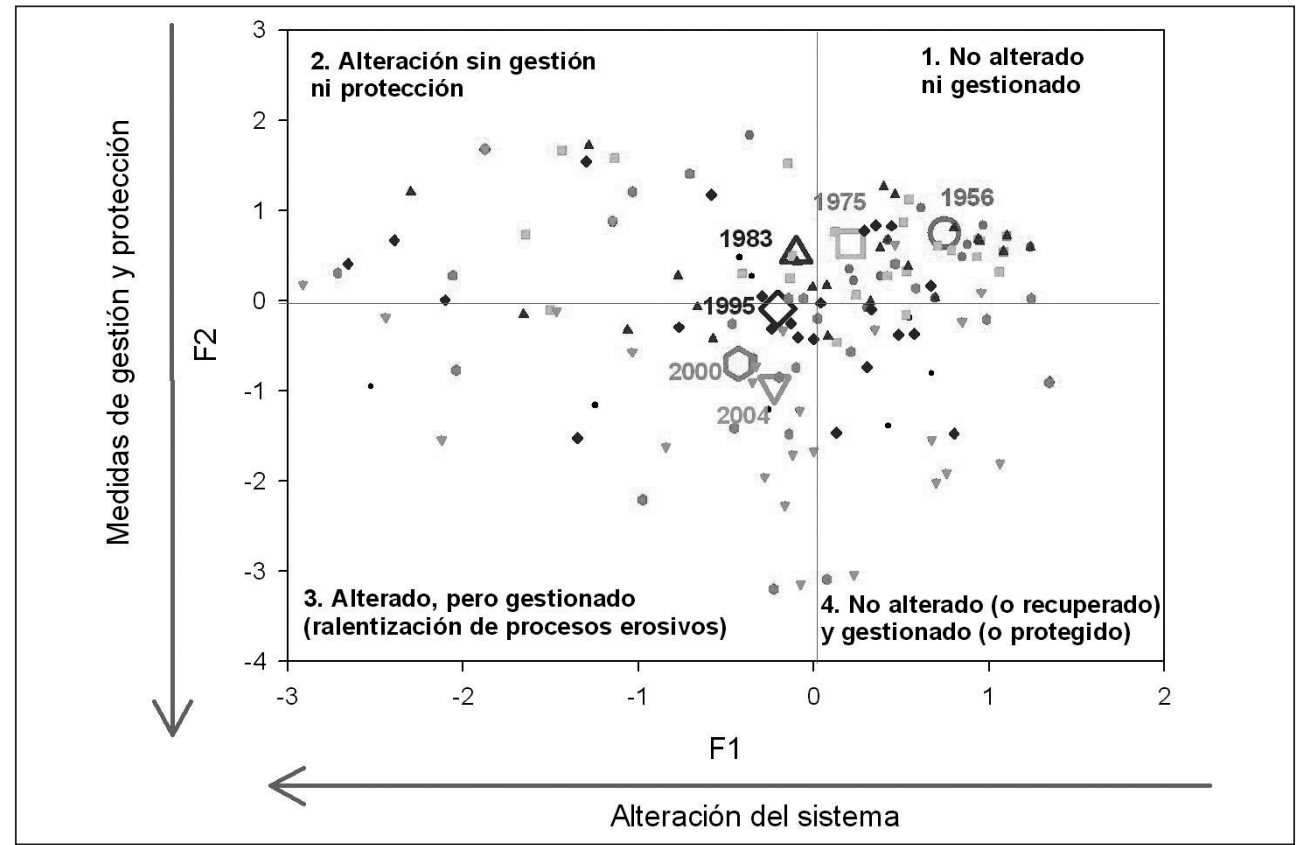

La representación de las medias anuales de los 28 sistemas dunares sobre el espacio factorial (Figura 3) indica como desde 1956 hasta 2000, en términos generales, se ha producido una alteración de los sistemas, especialmente acusada entre 1956 y 1995 . No obstante, se aprecia una trayectoria final hacia situaciones de recuperación debido a la aplicación de métodos de gestión que priorizan la recuperación de morfologías dunares. De este modo se deducen diferentes períodos que han afectado al conjunto de los sistemas playa-duna:

1. El periodo entre 1956 y 1983 coincide con la entrada de Menorca en el mercado turístico de Sol y playa, y la ocupación y consolidación de sus enclaves turísticos en su litoral, afectando esta ocupación los sistemas dunares. Partimos de una situación óptima de naturalidad de los sistemas dunares, hacia una alteración generalizada de estos debido a la falta de aplicación de medidas de gestión adecuadas, ni la presencia 
de figuras de gestión, entrando en una dinámica que tiende hacia la alteración y desaparición de formas dunares (se deduce de la reducción constante del valor F1 de los valores medios anuales de esta etapa). Se ha de tener en cuenta que durante 1969 se inauguró, en su emplazamiento actual, el nuevo aeropuerto de Menorca. Eso supuso un auténtico despegue turístico. El número de pasajeros que pasaron por el aeródromo se duplicó en sólo dos años. Frente a los 112.000 viajeros que utilizaban el antiguo aeródromo de Sant Lluís en 1968, se pasó a los 239.000 en 1970. Durante el año 2008 cuenta con cerca de 1.300.000 entradas de pasajeros al año (Murray et al., 2010).

2. Desde 1995 hasta 2000 , se aprecia una tendencia hacia una fase de alteración de los procesos de degradación de los sistemas dunares. Esta fase es progresivamente ralentizada debido al incremento en su gestión, ya sea por la entrada en vigor de figuras de protección y/o gestión (se atenúa de este modo el decrecimiento de los valores de F1).

3. El período comprendido entre el año 2000 y 2004 se aprecia una tendencia generalizada hacia la recuperación de los sistemas dunares, obteniendo una media más cercana al cuadrante inferior derecho, mediante la gestión y protección de estos. La aplicación de sistemas y métodos de gestión sostenibles, y la regulación del uso público sobre estas morfologías permite la estabilización de procesos erosivos de base y la recuperación de morfologías delanteras e interiores, foredune y dunas semiestabilizadas que habían sufrido procesos de reactivación, que contribuyen a la estabilización del conjunto del sistema dunar.

Es a partir del análisis de la evolución temporal de los 28 sistemas dunares se han podido identificar y definir cuatro comportamientos diferentes agrupados en tres grupos (Figura 4);

Grupo 1: Los sistemas no varían significativamente. Los valores anuales se mueven entre los dos cuadrantes definidos por el espacio factorial F1-F2 (superior y inferior). Dentro de este grupo identificamos dos subgrupos, que se mantienen estacionarios sin apenas variaciones interanuales (subgrupo 1a), y los que ligeramente empeoran y son objeto de medidas de gestión (subgrupo 1b). Tenemos un caso del tipo 1b: Escorxada, y 8 casos del subgrupo 1a: Trebalúger, Tamarells, Torreta, S'Enclusa, Morella, Presili, Son Saura del Sud y Es Bot, todos ellos sistemas dunares de tipología C. Estos sistemas situados en ANEI o Parque Natural, poco publicitados y con accesos siempre peatonales han sufrido leves procesos de degradación por su uso, y en menor medida por su gestión, muchas veces inexistente por la baja o nula explotación turística de estos arenales. De aquí que sus variaciones dentro del espacio factorial hayan sido condicionadas a su protección legal, ya que los sistemas presentan similares condiciones geoambientales que en el período 1956, pudiendo describirse en 2004 estadios 1 y 2 según Hesp (2002).

Grupo 2: Los sistemas que describen una trayectoria en forma de «C», es decir, los que empeoran a lo largo del tiempo pero son objeto de aplicación de medidas de gestión, muestran una cierta recuperación, observando dos tendencias de recuperación en función de la aceleración de estos sistemas a la respuesta ambiental de las medidas aplicadas. Esta recuperación de las formas dunares mediante procesos de recuperación sedimentaria puede derivar a hacia una tendencia hacia la naturalización del sistema en su conjunto, llegando en algunos casos a la situación de sistema recuperados, equiparándose a los del grupo 1 pero con elevados grados de uso y la aplicación de medidas de mantenimiento adecuadas para su correcta gestión. Tenemos 12 casos: Cala Pilar y Montgofre de tipología C, y que en estos 
casos presentan morfologías erosivas incipientes de base condicionada por el uso y agravada por cada una de las morfologías dunares remontantes. Cavalleria, Es Grau, Mitjana, Sa Mesquida, Algaiarens, S'Alairó, Son Bou B, Binimel·là, St. Tomàs y Tirant son sistemas dunares de tipología B, con elevados grados de frecuentación y publicitación que han sufrido en los últimos años importantes procesos de recuperación del frente dunar mediante la aplicación de diferentes técnicas de gestión paralelas y en diversos sectores del perfil playa-duna.

Grupo 3: Se trata de sistemas con clara vocación degenerativa y que su tendencia ha sido hacia la degradación continuada e incluso su desaparición aparente, convirtiéndose en superficies de deflación con morfologías relictuales aisladas y la neocolonización de morfologías asociadas a plantas pioneras que son erradicadas en períodos estivales al realizar actuaciones de adecuación de la playa para su uso turístico-recreativo. No muestran síntomas de recuperación, debido a la falta de gestión correcta. A pesar de todo, pueden ser objeto de medidas de gestión encaminadas a la recuperación de formas. Aparecen 7 sistemas: Macarelleta y Pregonda de tipologia B y con elevados índices de frecuentación, superiores a playas eminentemente urbanas, que no permiten la recuperación invernal de forma natural del sistema debido a la fuerte presión que sobre ellos se da. S'Olla, Punta Prima, Son Bou A, Cala Blanca y Arenal d'en Castell de tipología A y asociados directamente a sistemas urbanos turísticos (Roig-Munar y Comas, 2005).

Figura 4

IDENTIFICACIÓN DE TRES TIPOS DE EVOLUCIÓN TEMPORAL QUE MUESTRAN LOS SISTEMAS PLAYA-DUNA

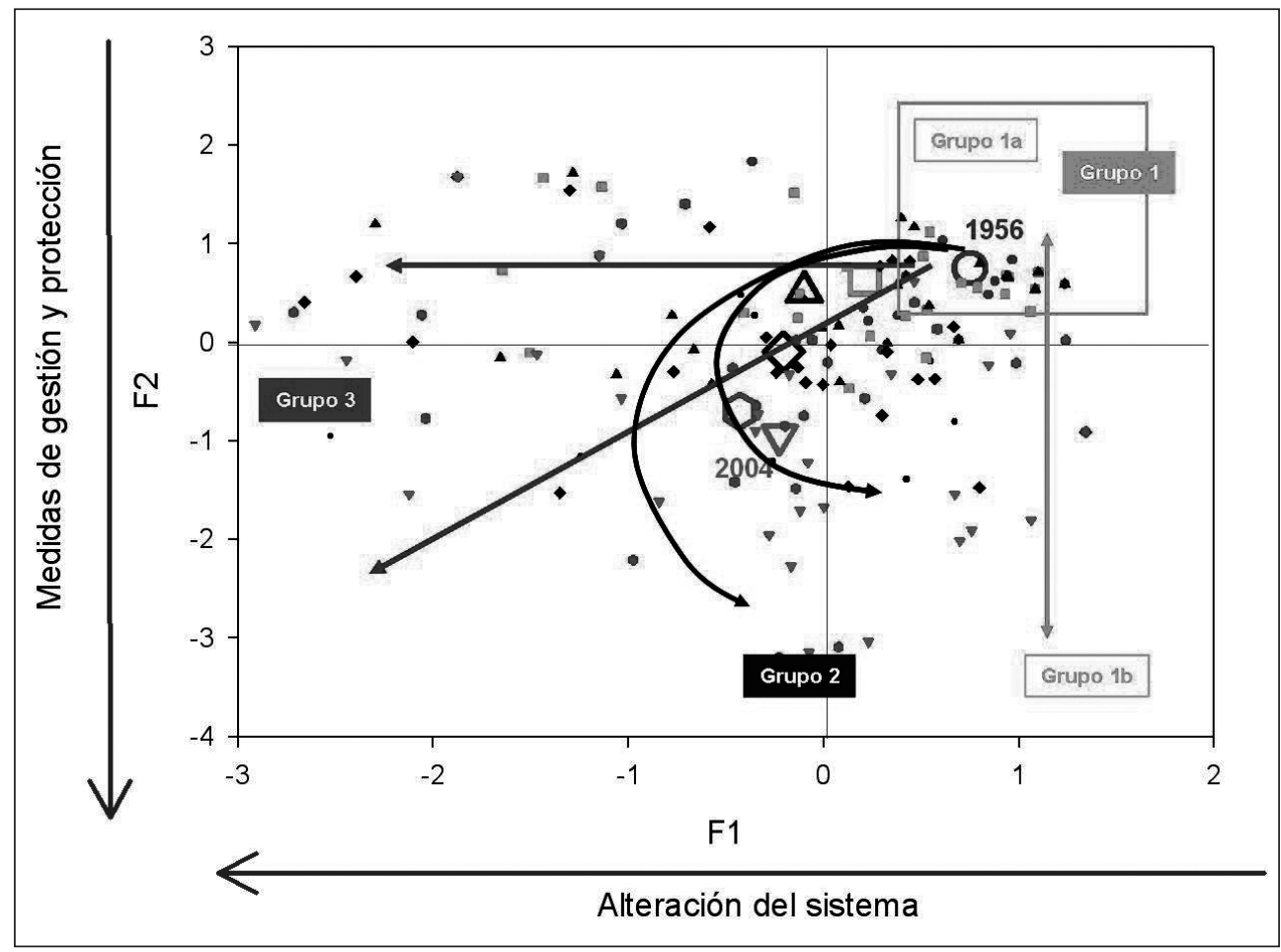


En términos particulares, sistema por sistema, ha habido diferentes evoluciones, desde sistemas dunares que se han mantenido estacionarios en su fase natural, sistemas que han sufrido constantes procesos de degradación hasta su práctica desestructuración y desaparición como sistema dunar, hasta aquellos que han revertido su tendencia degenerativa hacia la recuperación y naturalidad. Como término medio observamos sistemas altamente degradados que se han recuperado mediante la aplicación de medidas de gestión particularizadas a cada uno de estos sistemas y formas. La adopción de medidas de gestión, si son efectivas y basadas en el sistema natural como morfología, y no en el sistema natural como producto, han de redundar en una mejora, estabilización, recuperación y rehabilitación de la forma, y por ende de su cobertura vegetal. Así lo reflejan las evoluciones de los valores medios de F2-F3, que demuestran el crecimiento de cobertura vegetal entre 1995 y 2004, cobertura que se incrementa a lo largo de las formas erosionadas del sistema, por tanto demuestran una recuperación de formas que posteriormente son colonizadas vegetalmente dando lugar a la estabilización y mantenimiento del sistema en su conjunto.

La representación por separado de cada uno de los 28 sistemas analizados (Figura 5), permite trazar la evolución espacio-temporal que han seguido cada uno de ellos, relacionada directamente con su uso y gestión, condicionada en muchas ocasiones por su grado de publicitación. La tendencia generalizada ha sido hacia la degradación en sistemas dunares de tipología A y B, y estacionaria en sistemas dunares de tipología C. Estos últimos como Trebalúger, Es Bot, Tamarells o Sa Torreta demuestran este estacionamiento en los cuadrantes de no protegido y no alterada hacia el cuadrante de protegido. Otros casos como s'Arenal d'En Castell y Son Bou B presentan comportamientos paradigmáticos. En el primer caso no hay ningún tipo de gestión, produciéndose una degradación continuada del sistema, y dando como resultado morfologías relictas de primera línea. En el segundo caso, Son Bou B, la aplicación de medidas de gestión sostenibles, ha permitido revertir las tendencias de erosión observadas entre 1975 y 1983 hacia la recuperación de continuidad dunar entre los dos sistemas clasificados como A y B entre los períodos comprendidos entre 1995 a 2004.

De este modo, y basándonos en las tendencias observadas en los períodos de análisis, aportamos la propuesta de un modelo de evolución de sistemas playa-duna en el espaciotiempo, partiendo de las curvas de sensibilidad establecidas para tres sectores de los sistemas playa-duna de las islas Baleares (Brown y McLachlan, 1990, Roig et al, 2004, Roig y Martín, 2005) y usando la clasificación de los estadios de degradación de los sistemas dunares establecidos por Hesp (1998), y posteriormente modificados y ampliados por el mismo autor (2002). Así obtenemos, basándonos en el espacio factorial establecido en este trabajo, y los cuatro cuadrantes definidos en la Figura 3, las tendencias que pueden sufrir los sistemas dunares en función de su uso y gestión a lo largo del tiempo.

En la Figura 6 observamos cuatro escenarios tendenciales que pueden ser descritos como:

1. Estadio natural; el sistema presenta un funcionamiento morfodinámico natural, solo afectado en períodos puntuales por procesos erosivos e incluso acumulativos de origen natural. Estos procesos erosivos tienden a ser recuperados de forma sistemática por los propios mecanismos naturales del sistema. El resultado es un sistema playaduna bien estructurado sin interferencia e ingerencias de tipo antrópico. La popularización de estos espacios y su baja-media frecuentación pueden generar episodios puntuales de degradación que rápidamente pueden ser corregidos hacia su recupera- 
Figura 5

REPRESENTACIÓN EN EL ESPACIO FACTORIAL F1-F2 CON INDICACIÓN DE LOS VALORES ANUALES; 1956, 1975, 1983, 1995, 2000 Y 2004, DE LA EVOLUCIÓN ESPACIO-TEMPORAL DE LOS 28 SISTEMAS PLAYA-DUNA ANALIZADOS
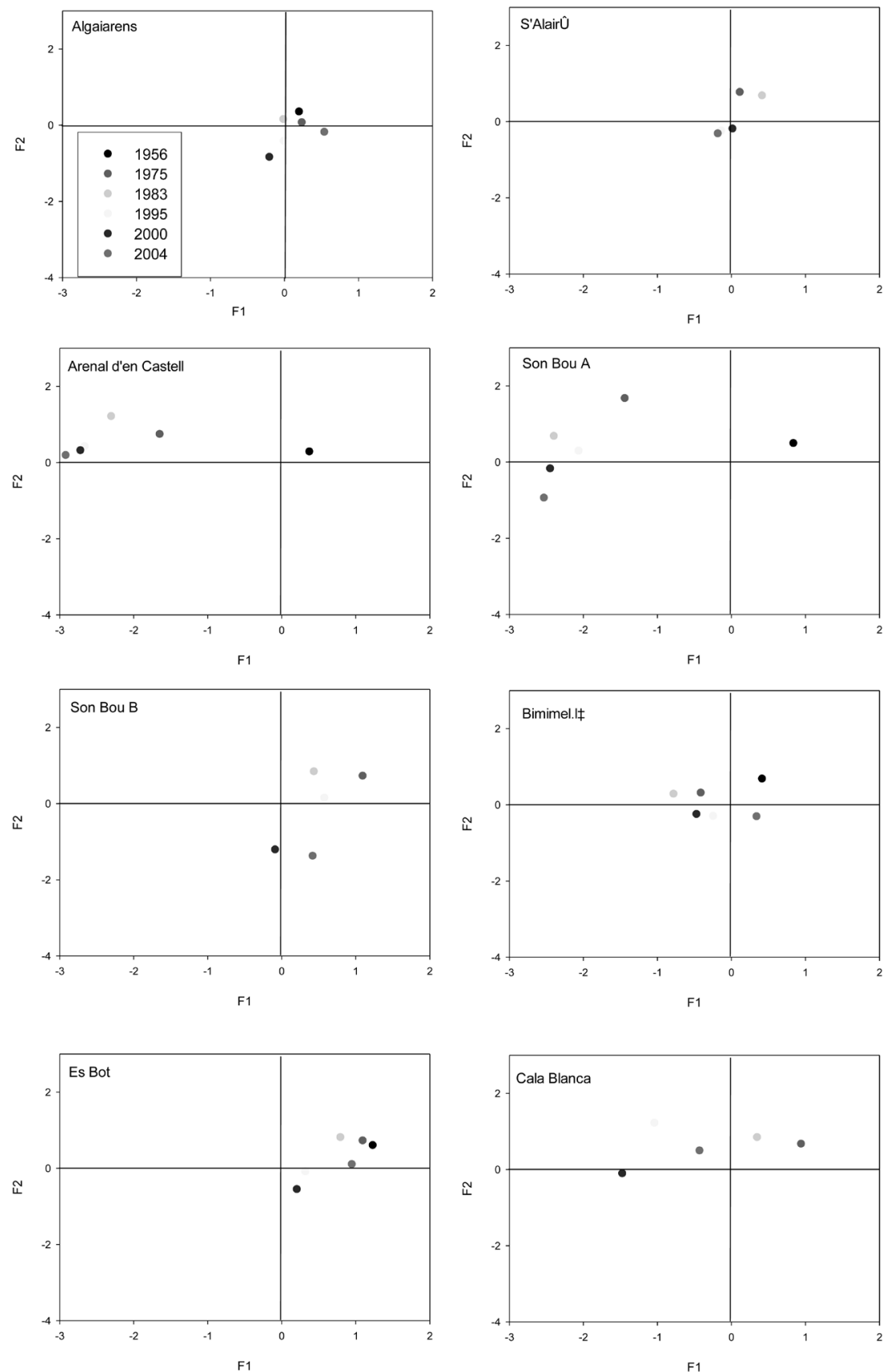

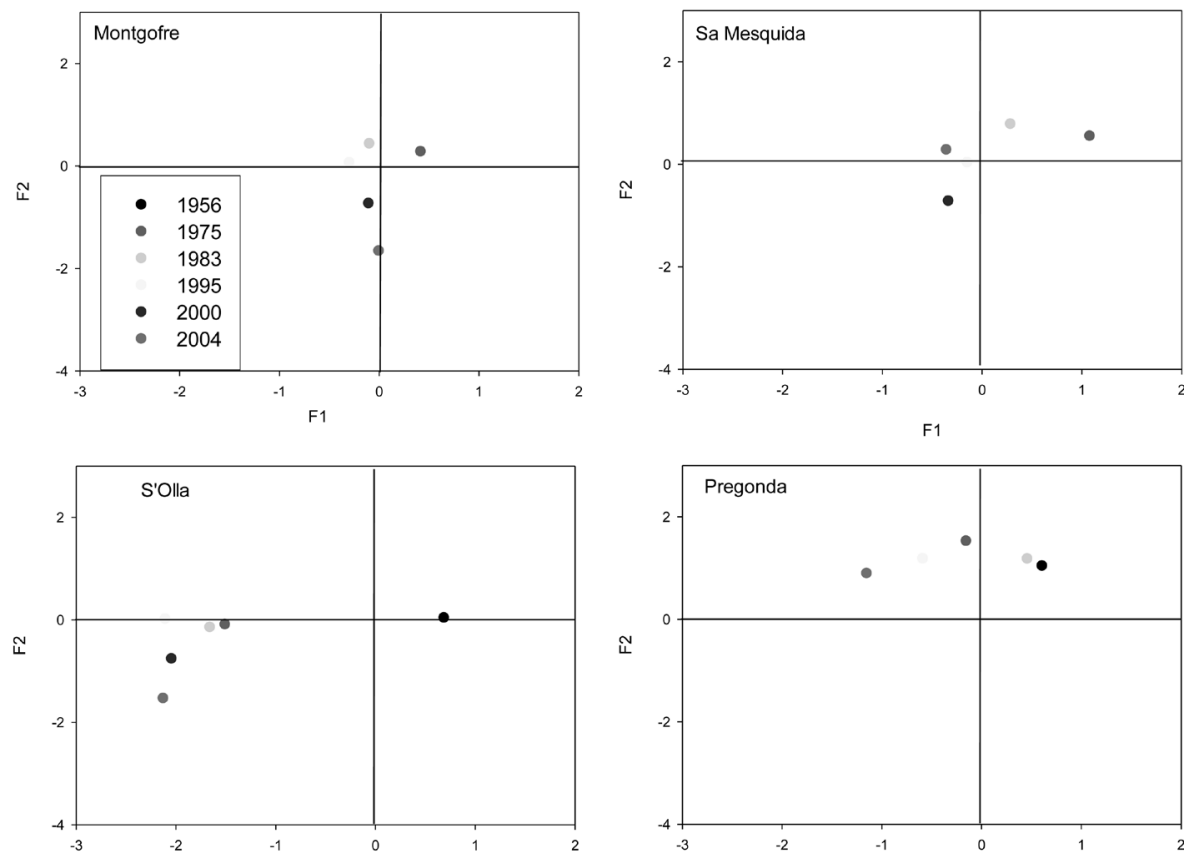

F1

F1
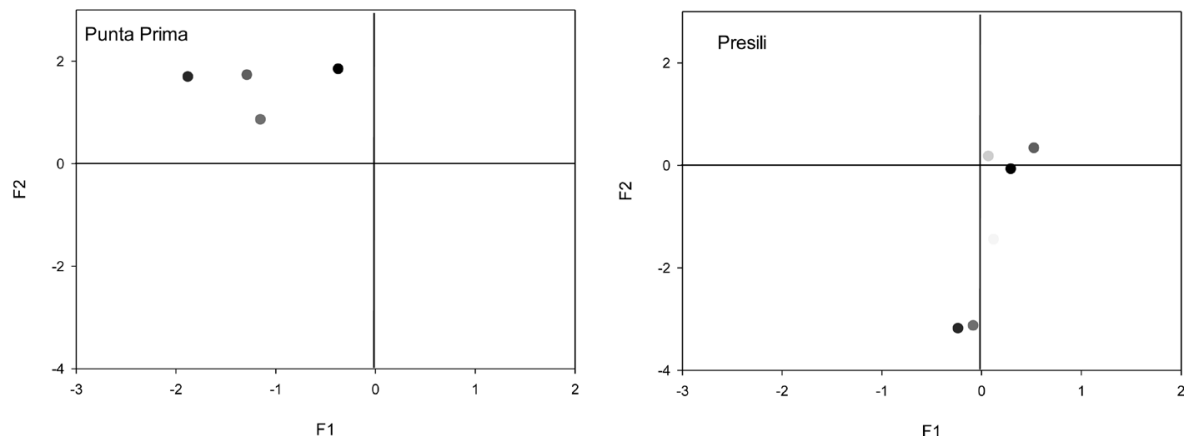

F1
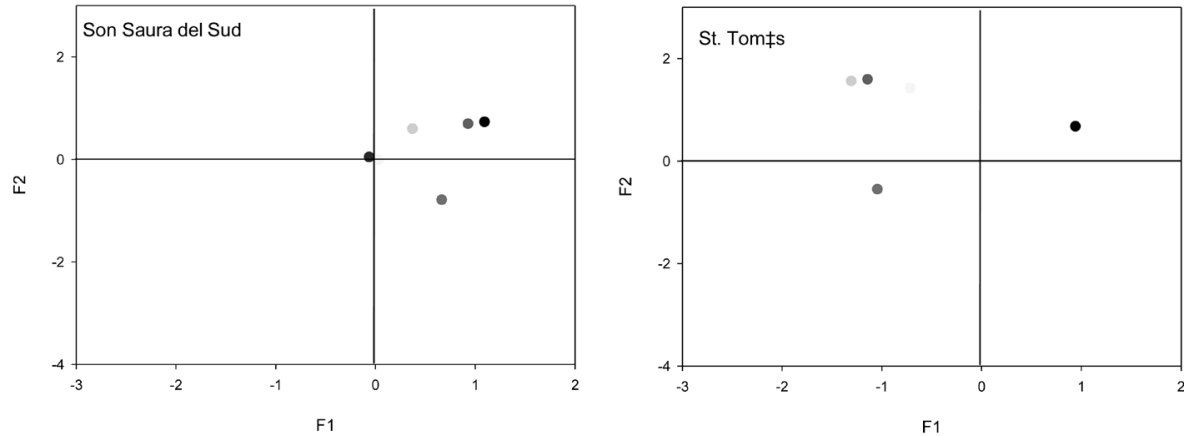

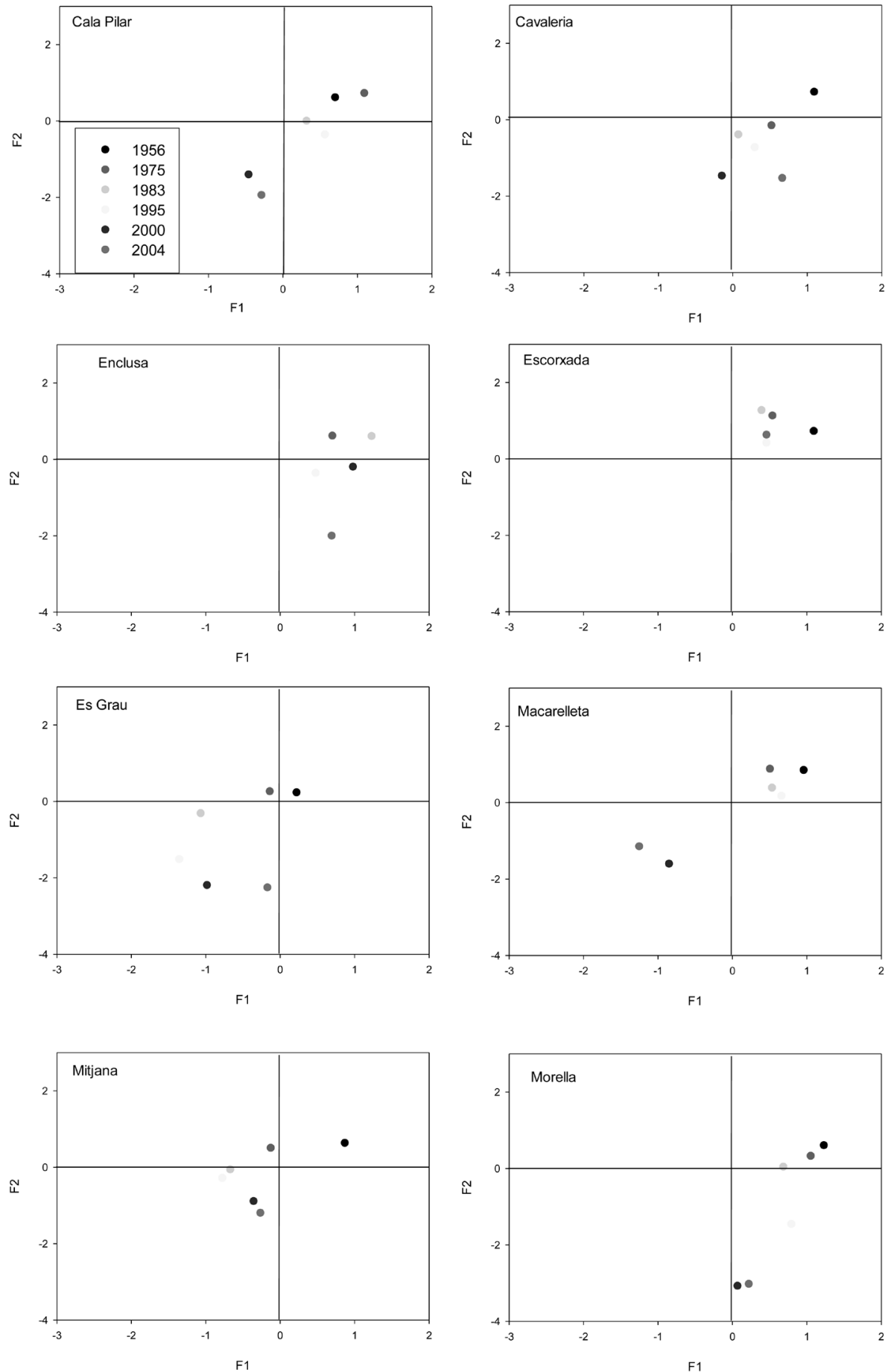

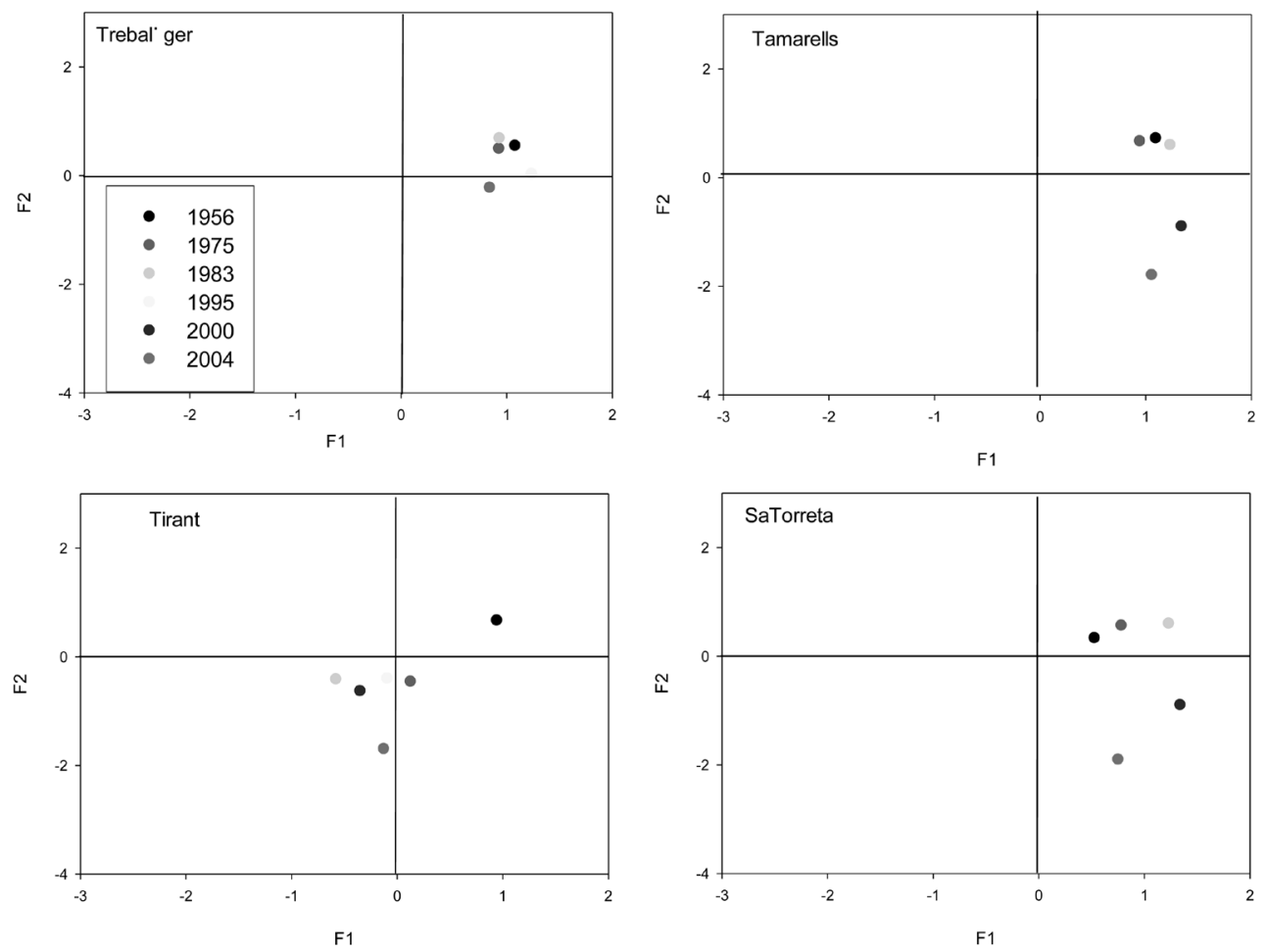

ción inicial, y manteniendo su perfil natural de playa-duna. Este estadio presenta un recorrido estacionario entre estado natural y de recuperación sin entrar en estadios de degradación.

2. Estadio de degradación; coincidiendo con la aparición de un turismo de Sol y playa, la popularización del litoral como espacio de recreación y recaudación, y el concepto eminentemente estático de los sistemas playa-duna, sin tener presente las morfologías dunares asociadas y su importancia como sistemas, estos sistemas empiezan a sufrir procesos de degradación de sus frentes dunares (vegetación y forma), ya sea por procesos de urbanización, ocupación mediante servicios o una nula o mala gestión. La afectación degenerativa sobre una de las curvas de sensibilidad del sistema dunar emergido, las foredunes, afecta al conjunto del sistema, con una tendencia que deriva hacia la desaparición de morfologías dunares delanteras, reactivación de morfologías interiores e importantes pérdidas de sedimento hacia el interior, en definitiva la creación de campos de delación sin morfologías asociadas que tienden a la desaparición del sistema.

3. Estadio de estabilización; podemos definir estos estadios de estabilización como estadios de máxima erosión que coinciden con la clasificación de los procesos erosivos definidos por Hesp (2002), o bien estadios de estabilización incipiente de morfologías dunares erosionadas, mediante la aplicación de medias de gestión acordes con el 
medio litoral arenoso. Los estadios de estabilización permiten trazar tendencias hacia la recuperación y mantenimiento del sistema delanteros que posteriormente afectara al sistema interior.

4. Estadio de recuperación; la aplicación de medidas correctoras en su uso y gestión permiten al sistema el recorrido inverso al seguido, pudiendo identificar la recuperación de formas dunares y continuidad de las mismas, así como la colonización de estas. La tendencia seguida por estos sistemas es en forma de «C».

Figura 6

PROPUESTA DE MODELO EVOLUTIVO ESPACIO-TEMPORAL DE LOS SISTEMAS PLAYA DUNA SEGÚN LA CLASIFICACIÓN MORFOECOLÓGICA DE HESP (2002)

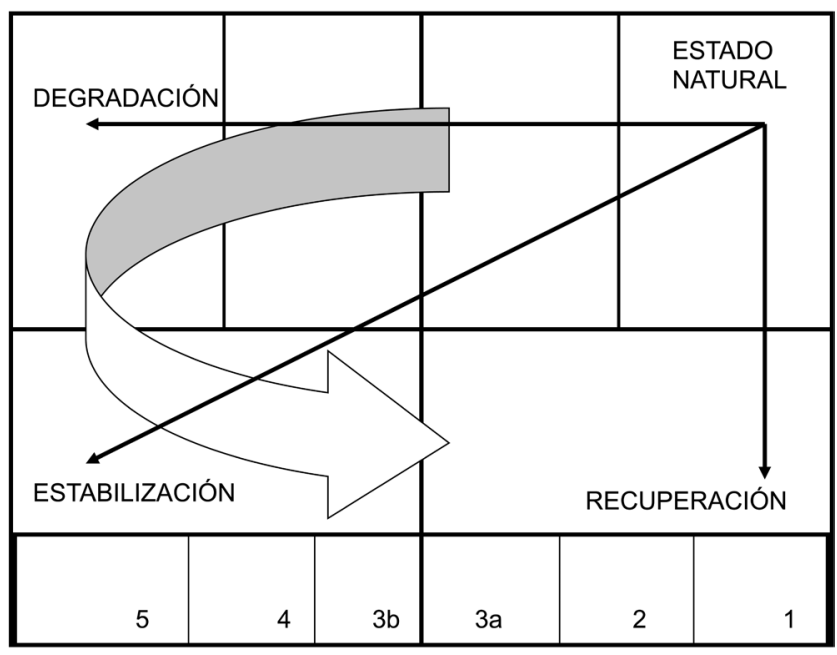

Clasificación HESP (modificada en el 3a i 3b)

\section{DISCUSIÓN}

El hecho de hacer funcionales los espacios litorales playa-duna para satisfacer la frecuentación masiva ha traído consigo serios problemas de conservación. Problemas como: alteración y eliminación de neomorfologías de playa-duna y su vegetación asociada, destrucción de procesos de formación de dunas, alteración y desestabilización de perfiles naturales de playa e incremento del transporte eólico por erosión de frentes dunares y mediante la reactivación de sistemas dunares semiestabilizados, pérdidas de diversidad biológica, pérdidas de superficies y volúmenes de playa, así como de sus morfologías asociadas, morfologías erosivas en primera y segunda línea, avance de lóbulos dunares, soterramiento de vegetación arbórea, entre otras.

En el caso de Menorca, donde el espacio costero arenoso se considera el espacio natural donde gravita la principal actividad económica de la isla, debido a su uso y aprovechamiento económico, valorado este mediante el método de valoración contingente en 33.532.156 €/ anuales, se requiere el uso de este espacio como recurso perdurable y sostenible sin altera- 
ción alguna a lo largo de décadas. Se precisa que los factores naturales y ambientales que posibilitan el desarrollo a lo largo del tiempo y la prestación de los servicios al consumidor turístico (empresa y/o usuario) proporcionen las expectativas de satisfacción a las expectativas y necesidades del usuario, siempre en consonancia con el estado geoambiental de los sistemas y con la declaración de Menorca Reserva de Biosfera. En este sentido el litoral en todo su conjunto (playas, calas y sistemas dunares) es el factor de producción de la industria turística de Menorca. Garantizar su permanencia en condiciones que satisfagan las demandas de los usuarios exige evitar procesos de degradación, aplicar medidas de mantenimiento, restauración, rehabilitación y recuperación.

El análisis de la evolución temporal de los sistemas dunares de Menorca refleja tres comportamientos diferenciales; sistemas estacionarios, sistemas recuperados gracias a la aplicación de medidas y sistemas de gestión, y sistemas degenerativos. La artificialización y las medidas de gestión son los factores que mejor definen la evolución temporal. Los procesos geomorfológicos de tipo erosivo se encuentran en la base de la evolución temporal observada.

De los tres comportamientos observados, el de los sistemas degenerativos es el de más interés para su análisis y gestión, debido a que estos reflejan, mediante procesos erosivos, las gestiones nulas o mas ineficaces que sobre ellos han actuado, así como las gestiones necesarias, comprobadas estas últimas con las tendencias observadas entre 2000 y 2004, mediante la aplicación de criterios geomorfológicos, ambientales y sociales.

Como en el caso de la clasificación de playas utilizada para la gestión litoral de arenales de Menorca e Islas Baleares (Roig-Munar y Comas, 2005), este análisis de la evolución espacio-temporal se presenta como una buena herramienta para medir y controlar el estado de conservación de los sistemas dunares.

\section{AGRADECIMIENTOS}

A Eulàlia Comas Lamarca por su ayuda en el trabajo de campo y tratamiento estadístico de datos.

A Macià Blázquez Salom del Departamento de Ciencias de la Tierra de la Universitat de les Illes Balears, por su ayuda y colaboración en la discusión de los criterios geomorfológicos y de gestión litoral de la isla de Menorca, así como por su ayuda en la gran diversidad de pruebas realizadas sobre los sistemas dunares para analizar sus comportamientos morfológicos.

\section{BIBLIOGRAFÍA}

ALONSO, J., ALCÁNTARA-CARRIÓ, J. y CABRERA, L. (2002): «Tourism resorts and their impacts on the beach erosion at sotavento beaches, Fuerteventura, Spain». Journal of Coastal Research, $\mathrm{n}^{\circ}$ 36, 1-7.

AMENGUAL, C., ARTIGUES, A.A., BAUZÁ VAN SLINGERLAND, A.; BLÁZQUEZ, M., BUADES, J.; CANALS, J., GINARD, X., GONZÁLEZ PÉREZ, J. M., MARTÍNEZ TABERNER, A., MOZO, I., PONS, G.X., PONS, A., RAMIS, M.M., RODRÍGUEZ PEREA, A., RULLAN SALAMANCA, O., VIDAL, M., VIVES, S. i VAN WALRE, N. 
(2010): «Els indicadors de sostenibilitat socioecològica de les Illes Balears (2003-2008)» en Observatori de Sostenibilitat i Territori (Murray, I., coord.). Palma de Mallorca, Grup d'Investigació sobre Sostenibilitat i Territori i Universitat de les Illes Balears.

BEJARANO, R., TEJADA, M. y JURADO, J. (1997): «Incidencia del actual proceso de intervención territorial en medios costeros de alto valor naturalístico» en Dinámica Litoral-Interior (I). Santiago de Compostela, Ed Universidade de Santiago de Compostela y AGE, 33-44.

BLÁZZUEZ, M. y ROIG, M. (1999): «L'abast de l'excursionisme a Mallorca». Boll. de Geografia Aplicada, $\mathrm{n}^{\circ}$ 1, 11-32.

BROWN, A.C. \& MCLACHLAN (1990): Ecology of sandy shores. US, Elsevier.

CARDONA, X., CARRERES, D., FRAGA, P. ROIG-MUNAR, F X, y ESTAÚN, I. (2004): «Avaluació de l'estat dels sistemas dunars de Menorca» en IV Jornades de Medi Ambient de les Illes Balears (Pons, G.X., coord.). Palma de Mallorca, SHNB, 309-311.

CROWELL, M., LEATHERMAN, S.P. y BUCKLEY, M. (1991): «Historical shoreline change: error analysis and mapping accuracy». Journal of Coastal Research, $\mathrm{n}^{\circ} 7$ (3), 839-852.

CURR, R. H., KOH, A., EDWARDS, E., WILLIAMS, A. T. y DAVIDES, P. (2000): «Assessing anthropogenic impact on Mediterranean sand dunes from aerial digital photography». Journal Coastal Conservation, $\mathrm{n}^{\circ}$ 6, 15-22.

GARCÍA, M., GALLEGO, J., WILLIAMS, A.T. y GARCÍA, F. (2001): «A coastal dune vulnerability classification. A case study of the SW Iberian Peninsula». Journal of Coastal Research, $\mathrm{n}^{\circ} 17$ (4), 802-811.

GARROTE, J. y GARZÓN, G. (2004): «Análisis del retroceso del frente de costa usando fotogramas aéreos, Oyambre (Cantabria)» en Procesos geomorfológicos y evolución costera (Blanco, R., López, J. y Pérez Alberti, A., coord.). Santiago de Compostela, Ed. Univ. Santiago de Compostela, $51-65$.

HESP, P. (1988): «Morphology, dynamics and internal stratification of some stablished foredunes in southeast Australia». Sedimentary Geology, n 55, 17-41.

HESP, P. (2002): «Foredunes and blowouts: initiation, geomorphology and dynamics». Geomorphology, $\mathrm{n}^{\circ} 48,245-268$.

LARANJEIRA, M. M, PEREIRA, A. y WILLIAMS, A. T. (1999): «Comparison of two checklist methods for assessment of coastal dune vulnerability». Bol. Inst. Esp. Ocea$n o g r, \mathrm{n}^{\circ} 15$ (1-4), 259-268.

LARSON, M. y KRAUS, N.C. (1995): «Prediction of creoss-shore sediment transportat different spatial and temporal scales». Marine Geology, $\mathrm{n}^{\circ}$ 126, 111-127.

LEATHERMAN, S.P. (1997): «Beach Rating: A Methodological Approach». Journal of Coastal Research, $\mathrm{n}^{\circ} 13$ (1), 253-258.

MARTÍN-PRIETO, J. A. y RODRÍGUEZ-PEREA, A. (1996): «Participación vegetal en la construcción de los sistemas dunares litorales de Mallorca» en IV Reunión de Geomorfología (Grandal d'Angade, A., coord.). O Castro (A Coruña), Sociedad española de Geomorfología, 785-798.

McLAUGHLIN, S., McKENNA, J. y COOPER, J.A. (2002): «Socio-economic data in coastal vulnerability indices: constraints and opportunities». Journal of Coastal Research, SI 36, 487-497. 
NORDSTROM, N. F. (1994): «Beaches and dunes of human altered coasts». Preogress in Physical Geography, no 18 (4), 497-516

PINTO, J. (2005): «El paisatge litoral». Espais, no 50, pp 66-77.

PYE, K (1982): «Morphological and desenvolpment of coastal dunes in a sumid tropical environment, Cape Bedford and cape Flattery, North Queensland». Geogr. Ann, ${ }^{\circ}{ }^{6}$, 213-227.

RITA, J., RODRÍGUEZ-PEREA, A. y TEBAR, J. (1988): Sistemas dunares de Menorca, valoración geoambiental y estado de la conservación. Documento inédito. Menorca, Institut Menorquí d'Estudis.

ROIG-MUNAR, F.X. (2004): «Análisis y consecuencias de la modificación artificial del perfil playa-duna por el efecto mecánico de su limpieza». Investigaciones Geográficas, $\mathrm{n}^{\mathrm{o}} 33,87-103$.

ROIG-MUNAR, F.X., y COMAS, E. (2005): «Propuesta de un modelo de clasificación para las playas de las islas Baleares mediante el análisis de variables de uso, estado y gestión». Boletín de la Asociación de Geógrafos Españoles, $\mathrm{n}^{\circ}$ 40, 429-448.

ROIG-MUNAR, F.X, RODRÍGUEZ-PEREA, A. y MARTÍN-PRIETO, J.A. (2004). «Influencia antrópica en la alteración del sistema playa-duna de Son Bou (Menorca)» en Contribuciones Recientes sobre Geomorfología (Benito, G. y Díez Herrero, A., coord.). Madrid, SEG-CSIC, 375-384.

SCHMITT, T. (1994): «Degradació de la vegetació psamòfila litoral de Mallorca». Boll. Soc. Hist. Nat. Balears, $\mathrm{n}^{\circ}$ 37, 151-174.

SERVERA, J (1997): Els sistemes dunars litorals de les Illes Balears. Tesi doctoral. Inédita. Palma de Mallorca, Universitat de les Illes Balears.

VALPREDA, E. y SIMEONI, U. (2003): «Assessment of coastal erosion susceptibility at the national scale: the Italian case». Journal of Coastal Conservation, $\mathrm{n}^{\circ} 9$ (1), 43-48.

VOIGT, A. y WEIDEMANN, G. (1997): Tourist paths-impacts on vegetation and soil fauna. An innovative approach to integrated dune management. Denmark, EUCC Dune seminar, 91-104.

WILLIAMS, A.T. y DAVIES, E. (1997): An innovative approach to integrated dune management. Denmark, EUCC Dune seminar, 150-157.

WILLIAMS, A.T. y MORGAN, R. (1995): «Beach awards and rating systems». Shore and Beach, no 63 (4), 29-33. 
